\title{
Scientific and Visual Effects Software Integration for the Visualization of a Chromatophore
}

\author{
Kalina Borkiewicz \\ Advanced Visualization Lab \\ National Center for \\ Supercomputing Applications \\ University of Illinois at Urbana- \\ Champaign \\ Urbana, IL, USA \\ kalina@illinois.edu \\ Robert Patterson \\ Advanced Visualization Lab \\ robertp@illinois.edu
}

\author{
AJ Christensen \\ Advanced Visualization Lab \\ National Center for \\ Supercomputing Applications \\ University of Illinois at Urbana- \\ Champaign \\ Urbana, IL, USA \\ achrist3@illinois.edu \\ Donna Cox \\ Advanced Visualization Lab \\ donnacox@illinois.edu
}

\author{
Stuart Levy \\ Advanced Visualization Lab \\ National Center for \\ Supercomputing Applications \\ University of Illinois at Urbana- \\ Champaign \\ Urbana, IL, USA \\ salevy@illinois.edu \\ Jeff Carpenter \\ Advanced Visualization Lab \\ jdcarpen@illinois.edu
}
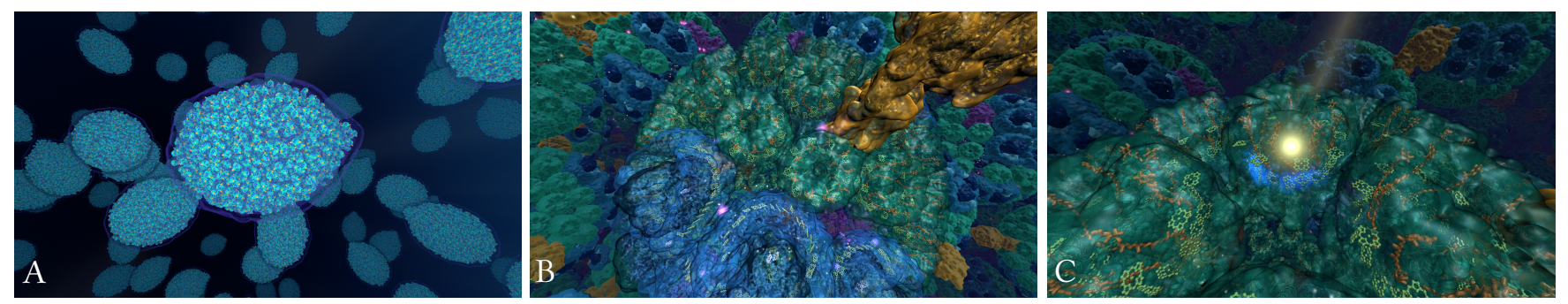

Figure 1: Stills from the chromatophore visualization. (A) depicts colony of purple bacteria, (B) shows the hero chromatophore, (C) depicts a photon hit and the beginning of an energy excitation.

\begin{abstract}
The scientific visualization of a chromatophore, a photosynthetic organelle, required the creation of a software integration pipeline to combine scientific software, visual effects tools, and custom camera choreography software. Furthermore, the rendering of this visualization in both fulldome and 4K3D format was done using a custom supercomputer rendering pipeline.
\end{abstract}

\section{CCS CONCEPTS}

- Human-centered computing $\rightarrow$ Scientific Visualization

\section{ACM Reference format:}

Kalina Borkiewicz, AJ Christensen, Stuart Levy, Robert Patterson, Donna Cox, and Jeff Carpenter. 2018. Scientific and Visual Effects Software Integration for the Visualization of a Chromatophore. In Proceedings of SIGGRAPH Asia 2018 Posters, Tokyo, Japan.

Permission to make digital or hard copies of part or all of this work for personal or classroom use is granted without fee provided that copies are not made or distributed for profit or commercial advantage and that copies bear this notice and the full citation on the first page. Copyrights for third-party components of this work must be honored. For all other uses, contact the Owner/Author. Copyright is held by the owner/author(s).

SA'18 Posters, December 04-07, 2018, Tokyo, Japan

ACM 978-1-4503-6063-0/18/12.

$10.1145 / 3283289.3283324$

\section{Introduction}

The Advanced Visualization Lab (AVL) is a research team that specializes in creating production-quality cinematic scientific visualizations of supercomputer simulations for public outreach. The AVL worked with the Theoretical and Computational Biophysics Group (TCBG) to a visualize a simulated chromatophore and its environmental context, for an upcoming fulldome show titled "Birth of Planet Earth". A chromatophore is an early photosynthetic organelle and a predecessor to chloroplasts found in modern plant cells. This visualization helps to describe how light is turned into chemical energy through the quantum dynamics of organic molecules.

The challenge in creating a cinematic visualization based on real scientific data is that no single piece of software can both (1) read scientific data formats and (2) provide artistic tools and controls for lighting, shading, materials, designed effects, and camera choreography. Visual effects tools excel at creating cinematic imagery, whereas scientific tools excel at reading complex scientific data formats and visualizing them for purposes of data analysis. In order to visualize the chromatophore in an engaging way for the general public, it was necessary to develop a pipeline which integrates both categories of tools. 


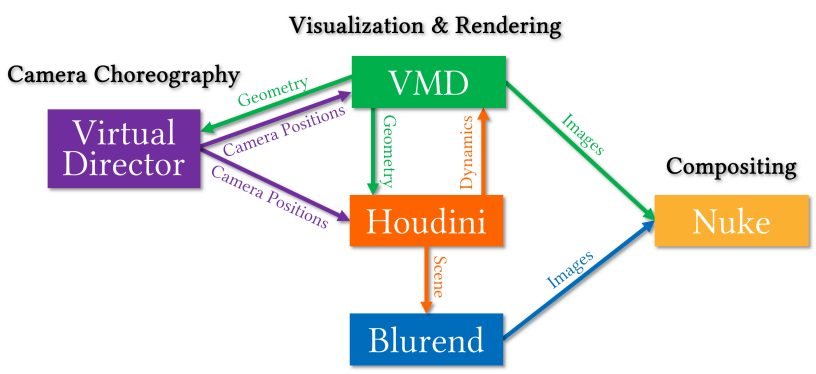

Figure 2: Software integration pipeline involving custom software Virtual Director and Blurend, scientific software VMD, and visual effects software Houdini and Nuke.

\section{Technical Approach}

The visualized chromatophore data came from a 60TB, 5 million atom computational simulation run by the TCBG [Stone et al. 2016] on the Blue Waters and Titan supercomputers. Visualizing the energy harvesting process of a photosynthetic purple bacterium [Cartron et al. 2014] involved combining structural models from atomic, protein, organelle, and cell scales using multiple software tools.

The TCBG team that produced the chromatophore simulation also created VMD [Humphrey et al. 1996], a software that offers a rich set of tools for molecular visualization. This was the starting point for the AVL in creating this visualization. However, it quickly became apparent that, although incredibly efficient in creating molecular imagery, VMD did not have the artistic authoring and effects capabilities necessary for contextualization of the simulation and illustration of non-molecular dynamic elements.

The visual effects software, Houdini, provided a good platform for designing a programmed choreography of dynamic elements. First, photons of light had to be made to arrive from an infinite distance and collide with a specific protein. Next, a guided random walk of energy bundles was coded to occur in front of the camera. A series of proton and quinol sprites was made to migrate, moving seemingly at random using Brownian motion, but with timing synchronized with energy transfer events, and following paths that passed through designated points on the chromatophore where those events take place. The path-generating tool avoided collisions with protein structures. Finally, the effects were duplicated across the "hero" organelle, which was instanced thousands of times in the scene to suggest the biological complexity of a cluster of bacteria.

To link the two pieces of software, AVL needed a way to share camera views and positions. AVL's pipeline for designing cinematic animations includes Virtual Director [Lascara et. Al 1999], in-house software for camera choreography, in which a 3D mouse can be used to fly organically around an object to identify scientifically important features and synchronize the timing of the camera to specific events. VMD exported subsets of a chromatophore model into a form which Virtual Director could display. To see how the VMD-rendered chromatophore would look from a chosen point of view, AVL extended Virtual Director to export its current viewpoint via a network socket, and used VMD's Tcl-based scripting to translate camera positions into its terms. This allowed for a live connection between Virtual Director and VMD sessions, with the VMD view synchronized to the Virtual Director view. A batch rendering pipeline was built, accepting a complete Virtual Director camera path and driving VMD to record each frame of animation.

The plan to present the energy chain included showing electronic excitations within the chromatophore. These elements were embedded in transparent surfaces rendered by VMD, so we needed VMD to render these as well, but this was not a natively-supported feature. John Stone, the primary developer of VMD at TCBG, adapted VMD so that AVL could add a "glow" shader to its shader library. The blue-glow excitations, and embedded protons and quinol elements, use this shader.

AVL rendered numerous layers in Houdini along with depth passes to control relative luminance levels and depth effects. The Houdini scene rendering was done on the Blue Waters supercomputer at the University of Illinois at Urbana-Champaign with AVL's custom supercomputer rendering pipeline called Blurend and a custom dome lens shader for the fulldome imagery. Rendering on Blue Waters allowed us to iterate on different treatments quickly and to render many more images at once than we could on our local cluster, rendering as many as 20,870 image frames over the course of one weekend. It also allowed us to push the limits of how much geometry could be represented in a scene - in this case $3001.3 \mathrm{~GB}$ volumetric bacteria membranes containing more than one million $900 \mathrm{MB}$ chromatophore surface geometries. The scene consists of 14 render layers from both Houdini and VMD composited with Nuke, where the scene was made to appear integrated and under water.

\section{Conclusion and Future Work}

An effective scientific visualization is understandable, educational, and inspiring to broad audiences. We will be working with psychology and educational research collaborators in conducting audience testing and evaluation to measure the effectiveness of this visualization. Based on feedback from these studies, we may make modifications to the final visualization and narration script. It is important to validate our design decisions through this feedback process.

\section{ACKNOWLEDGMENTS}

This visualization is partially funded by NSF grant ACI-1445176.

\section{REFERENCES}

Michaël L. Cartron, John D. Olsen, Melih Sener, Philip J. Jackson, Amanda A. Brindley, Pu Qian, Mark J. Dickman, Graham J. Leggett, Klaus Schulten, and C. Neil Hunter 2014. Integration of energy and electron transfer processes in the photosynthetic membrane of Rhodobacter sphaeroides. Biochimica et Biophysica Acta (BBA) Bioenergetics 1837, 10 (2014), $1769-1780$.

William Humphrey, Andrew Dalke, and Klaus Schulten. 1996. VMD: Visual molecular dynamics. Journal of Molecular Graphics 14, 1 (1996), 33 - 38

Cathy M. Lascara, Glen H. Wheless, Donna Cox, Robert Patterson, Andrew Johson, Jason Leigh, and Ahbinov Kapoor. 1999. TeleImmersive Virtual Environments for Collaborative Knowledge Discovery. In Advanced Simulation Technologies Conference. 11-15.

John E. Stone, Melih Sener, Kirby L. Vandivort, Angela Barragan, Abhishek Singharoy,Ivan Teo, João V. Ribeiro, Barry Isralewitz, Bo Liu, Boon Chong Goh, James C. Phillips, Craig MacGregor-Chatwin, Matthew P. Johnson, Lena F. Kourkoutis, C. Neil Hunter, and Klaus Schulten. 2016. Atomic detail visualization of photosynthetic membranes with GPU-accelerated ray tracing. Parallel Computing. 55 (2016), $17-27$. 